\title{
Effect of a Counterirritant on Pain and Restricted Range of Motion Associated With
} Delayed Onset Muscle Soreness

\author{
By: Scott C. Haynes and David H. Perrin ${ }^{1}$
}

Haynes, S.C. \& Perrin, D.H. (1992). Effect of a counterirritant on pain and restricted range of motion associated with delayed onset muscle soreness. Journal of Sport Rehabilitation, 1, 13-18.

\section{***Note: Figures may be missing for this format of the document ***Note: Footnotes and endnotes indicated with parentheses}

\section{Abstract:}

This investigation examined the effect of a counterirritant on delayed onset muscle soreness (DOMS). Fourteen female subjects had DOMS induced in the elbow flexor muscles of the nondominant arm through repeated eccentric contractions. Subjects returned $48 \mathrm{hrs}$ after exercise and were randomly assigned to either a counterirritant ointment (CO) or placebo ointment (PO) treatment group. They were asked to quantify the amount of pain they experienced using a graphic pain rating scale while attempting to extend their elbow. Eight $\mathrm{ml}$ of the counterirritant or placebo ointment was applied to the anterior aspect of the arm centered directly over the biceps brachii. Pain and range of motion measurements were taken both pretreatment and 15 minutes posttreatment. Analysis of variance revealed that the $\mathrm{CO}$ group experienced significant pain relief and increased range of motion while the placebo group showed no significant changes. These findings suggest that counterirritants may be an effective means of treating the pain and restricted range of motion associated with delayed onset muscle soreness.

\section{Article:}

A wide variety of therapeutic agents are used to relieve the pain and stiffness associated with injury to the musculoskeletal system. Counterirritants, often called sports balms or analgesic creams, are one popular form of treatment that have been used for decades. In 1988 over $\$ 133$ million was spent on such products (2).

The Food and Drug Administration has classified counterirritants as a Category 1 drug. This means counterirritants are considered safe and effective in the treatment of muscle soreness (5). However, the exact physiological mechanisms for the pain relief are not known (2). In addition, minimal research has been conducted to assess the effectiveness of counterirritants on reducing pain. As such, the purpose of this investigation was to examine the effects of a counterirritant on the pain and loss of motion associated with delayed onset muscle soreness.

\section{SUBJECTS AND DESIGN}

\footnotetext{
${ }^{1}$ At the time of this study both authors were with the Curry School of Education at the University of Virginia, Charlottesville, VA 22903. Scott Haynes is now with the Physical Therapy Program, University of Delaware, Newark, DE 19716.
} 
Fourteen female subjects (age $19.4 \pm .93 \mathrm{yrs}$, ht $166.20 \pm 5.95 \mathrm{~cm}$, wt $61.10 \pm 7.31 \mathrm{~kg}$ ) were recruited to participate in the study. The method of inducing DOMS was explained to them prior to their participation. All subjects read and signed an informed consent form approved by a university human investigations committee. Only those subjects who were free from recent weight training activities within the last 6 months and had no history of upper extremity injury were allowed to participate. They were instructed to refrain from using analgesic and antiinflammatory medications, physical therapy, and extensive upper arm exercise until after the effects of the treatment had been assessed.

During the first of two test sessions, initial range of motion and pain values were measured and then delayed onset muscle soreness was induced. During the second session ( $48 \mathrm{hrs} \mathrm{later),} \mathrm{range} \mathrm{of} \mathrm{motion} \mathrm{and}$ pain values were again measured. Subjects were then randomly assigned to receive either a counterirritant treatment $(\mathrm{CO})$ or a placebo ointment treatment (PO). Fifteen minutes after application of the counterirritant or placebo treatment, range of motion and pain values were again measured in the same order.

\section{ASSESSMENT OF MOTION}

Elbow extension was measured by one researcher using a standard metal goniometer. The axis approximating the center of rotation of the humero-ulnar articulation and the bisection of the arm and forearm were used as landmarks. Each subject was seated in a chair and positioned with her nondominant elbow resting on the end of the table and her shoulder in approximately $75^{\circ}$ of flexion. She was asked to extend the elbow as far as possible. Two measurements of elbow extension were obtained and the average of the two were used for data analysis.

\section{ASSESSMENT OF PAIN}

The intensity of perceived pain associated with DOMS was measured using a graphic pain rating scale. The scale used in this study was one developed by Denegar $(3,4)$. It is similar to the scale used in previous studies involving delayed onset muscle soreness $(3,4)$ and is based on a previous verbal descriptive scale designed by Talag (12). The scale was a 12-cm horizontal line with "no pain" on the far left and "unbearable pain" on the extreme right. The following descriptors were spread continuously along the line from left to right: "dull ache," "slight pain," "more slight pain," "painful," and "very painful" (Figure 1). Pain was quantified by measuring the length of the line from the extreme left of the scale to the subject's mark. Values were rounded to the nearest half-centimeter. The subjects were asked to rate the pain they experienced while attempting to extend their arm. Pain measurements were always measured after range of motion measurements were taken.

NO

UNBEARABLE

PAIN DULL ACHE SLIGHT PAIN MORE SLIGHT PAIN PAINFUL VERY PAINFUL PAIN

Figure 1-Graphic pain rating scale.

\section{DELAYED ONSET OF MUSCLE SORENESS (DOMS)}

Repeated eccentric muscle contractions were used to induce delayed onset muscle soreness. This lifting protocol has previously been used to induce DOMS in the nondominant arm by various researchers $(3,4)$. 
Subjects were instructed to repeatedly lower a 25-lb dumbbell from full elbow flexion to complete extension over a 2-second time period. To avoid concentric contractions by the subjects, the researcher lifted the weight between eccentric contractions. When the subjects were unable to control the weight for 2 seconds, the weight was decreased to $20 \mathrm{lbs}$. This process was continued in 5-lb. decrements until the subject was unable to control $5 \mathrm{lbs}$ or complete 30 repetitions with $5 \mathrm{lbs}$.

\section{TREATMENT}

For application of counterirritant ointment (Cramergesic $®$, Cramer Products, Inc., Gardner, KS) or placebo ointment, a rectangular stencil measuring $12 \mathrm{~cm}$ x $15 \mathrm{~cm}$ (180 square $\mathrm{cm})$ was centered directly over the biceps brachii with the longer axis in line with the cubital fossa and the deltopectoral groove. The ointment was applied to the skin with a wooden tongue blade to minimize any massage effects.

The subjects were randomly divided into two groups for application of $8 \mathrm{ml}$ of ointment. During the second session, one group received the counterirritant ointment $(\mathrm{CO})$ and the other group received a placebo ointment (PO) of similar consistency and having no physiological effects.

Two 2 x 2 analysis of variances (Group x Time) were computed for the pain and range of motion (ROM) data, respectively.

\section{RESULTS}

The ANOVA for range of motion revealed a significant measurement-time-by-treatment interaction, $\mathrm{F}(1,2)=14.11, \mathrm{p}<0.05$. The counterirritant ointment group increased in $\mathrm{ROM}$ (pre $=8.72^{\circ}$ from full extension, post $=2.71^{\circ}$ from full extension) while the placebo ointment group showed no significant change (pre $=11.93^{\circ}$ from full extension, post $=10.50^{\circ}$ from full extension) in ROM assessment (Figure 2). The ANOVA for pain also revealed a significant measurement-time-by-treatment interaction, $F(1,12)$ $=7.02, \mathrm{p}<0.05$. The counterirritant ointment group reported a decrease in perceived pain $(\mathrm{pre}=6.43 \mathrm{~cm}$, post $=4.50 \mathrm{~cm}$ ) while the placebo ointment group demonstrated a nonsignificant change $($ pre $=6.29 \mathrm{~cm}$, post $=5.79 \mathrm{~cm}$ ) in perceived pain (Figure 3 ).

\section{DISCUSSION}

The major finDing of this investigation was that a counterirritant treatment decreased the perceived pain and decreased the restriction in elbow extension range of motion associated with DOMS. This finding is consistent with what is observed empirically with the use of counterirritants. Generally most athletes use counterirritants on minor musculoskeletal injuries for the mild analgesic effect. This mild analgesic effect inhibits the painful stimuli and consequently allows for greater pain-free range of motion.

Pain measurement is a necessity in the research of therapeutic agents and pain control mechanisms. However, this task is quite difficult because pain is a 


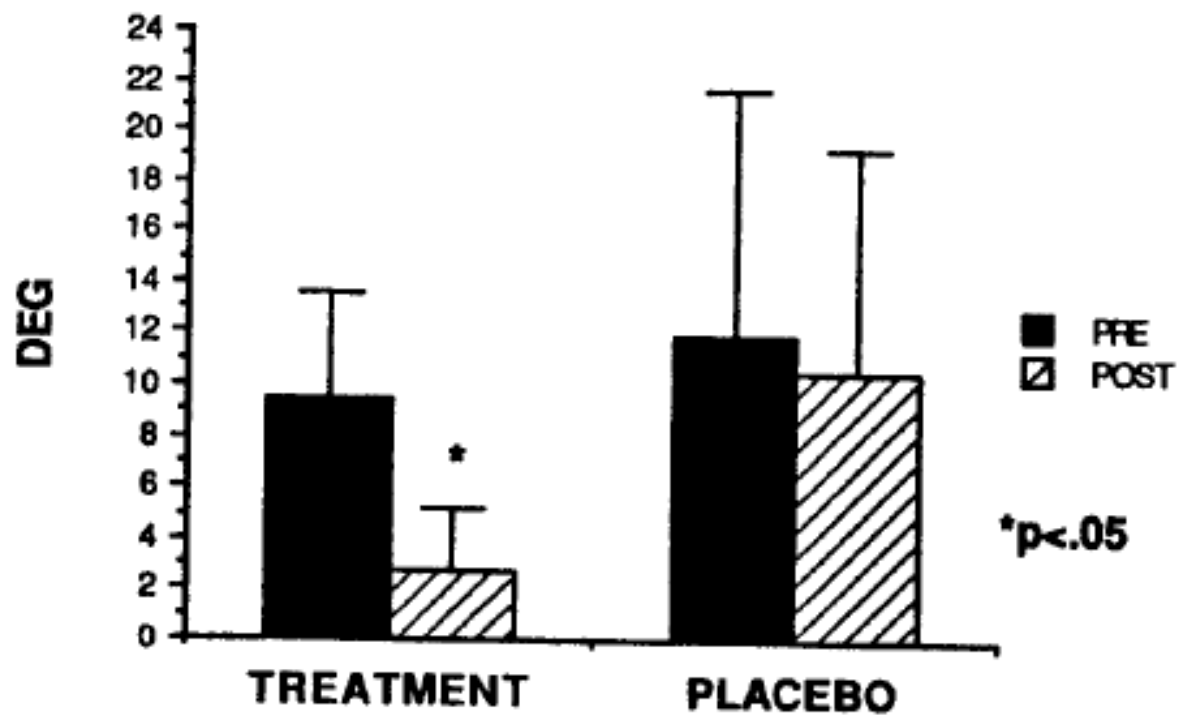

Figure 2--- Elbow range of motion expressed as degrees from full extension

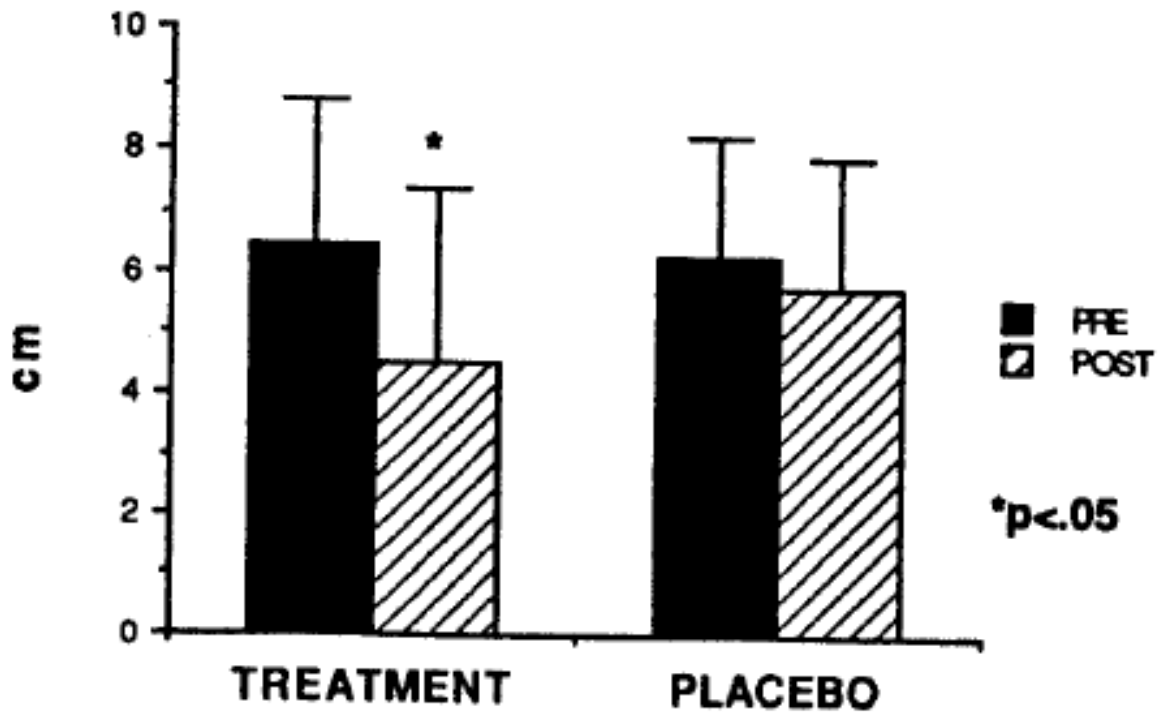

Figure 3 - Perceived pain during elbow extension.

multidimensional phenomenon (9). Melzack and Wall discussed the importance of construct validity in pain assessment and stressed that the instrument should only measure one construct and therefore should be unidimensional. Pain rating scales are often used to measure pain. Simple pain rating scales only measure the intensity dimension of pain (9).

A variety of pain scales have been used to measure pain intensity. Three commonly used types are verbal descriptive scales, visual analogue scales, and graphic pain rating scales (7). Verbal descriptive scales consist of a list of adjectives that describe different levels of pain. These descriptors are typically placed 
in numeric order with "no pain" being at zero and "unbearable pain" at some given number. The validity of verbal descriptive pain scales has been clinically tested and shown to be valid in defining the action of analgesics (8). Visual analogue scales consist of a line with the extremes of pain on each end. For example, "no pain" and "unbearable pain" may appear on the ends of the line. The scale is scored by measuring the length of the line from "no pain" to the mark made by the subject. Huskisson (6) has shown that visual analogue scales are sensitive and reproducible methods of expressing pain severity.

Graphic pain rating scales are a composite of the two previously mentioned scales. These scales have extreme descriptors at the ends of a line with descriptive terms spread out along the line. Scott and Huskisson (10) reported a uniform distribution of scores with no tendency for scores to cluster together in graphic pain rating scales when compared to other types of pain scales.

Delayed onset muscle soreness was chosen to exemplify the conditions of soft tissue injuries following athletic trauma. These musculoskeletal pathologies are commonly characterized by an increase in pain and a decrease in joint range of motion. DOMS is typically induced by repetitive eccentric loading of a muscle. Subsequent soreness and stiffness typically begins 12 to 24 hours after the exercise bout and steadily increases for the first 24 to 48 hours. The symptoms will gradually decrease between 48 and 72 hours and eventually disappear in 5 to 7 days (1). The DOMS protocol used was found to be an excellent laboratory model for replicating the signs and symptoms of athletic soft tissue trauma.

Other researchers have looked at the effects of counterirritants. Shellock (11) reported that topical application of a counterirritant produced a three- to fourfold increase in skin blood flow, which was sustained for at least 1 hour. White and Sage (13) studied the effects of counterirritants on induced soreness in the forearms. Electromyographic readings taken before and 48 hours after exercise showed that counterirritants significantly lowered muscle action potentials when compared to a placebo. In a subsequent study, White and Sage (14) studied the effects of counterirritants on arthritic patients. Again, electromyographic readings showed a decrease in muscle action potential when a counterirritant was applied to the sore joint. However, the clinical significance of these findings was not discussed.

Although this investigation demonstrated a decrease in pain and an increase in range of motion, further research should examine the duration of the effects of a counterirritant. The treatment protocol used in this study consisted of waiting 15 minutes before the pain and range of motion measurements were reassessed. This length of time was chosen because pilot study results showed a peak "warming" sensation from the counterirritant at this time. Additionally, further research should compare the effectiveness of the plethora of counterirritant brands on the market.

\section{CONCLUSION}

The counterirritant applied in this study resulted in decreased perceived pain and increased elbow extension range of motion. These data suggest that counterirritants may have an important clinical role in the treatment of minor athletic injuries. Because many athletes complain of increased pain and decreased range of motion (stiffness) when performing rehabilitative exercises, counterirritants may be employed to make the athlete less hesitant about performing therapeutic exercises.

\section{REFERENCES}

1. Abraham, W.J. Factors in delayed muscle soreness. Med. Sci. Sports Ex. 9:11-20, 1977.

2. Barone, J. Topical analgesics: How effective are they? Phys. Sportsmed. 17:163-168, 1989. 
3. Denegar, C.R., and C.B. Huff. High and low frequency TENS in the treatment of musculoskeletal pain: A comparison study. Athl. Training 23:235-237, 1988.

4. Denegar, C.R., D.H. Perrin, A.D. Rogol, and R. Rutt. Influence of transcutaneous electrical nerve stimulation on pain, range of motion, and serum cortisol concentration in females experiencing delayed onset muscle soreness. J. Orthop. Sports Phys. Ther. 11:100-103, 1989.

5. Federal Register. External analgesic drug products for over-the-counter human use. 48:5825-5869, 1983.

6. Huskisson, E.C. Visual analogue scales. In: Pain Measurement and Assessment, R. Melzack (Ed.). New York: Raven Press, 1983.

7. Keele, K.D. The pain chart. The Lancet. July 3:6-8, 1948.

8. Melzack, R. Concepts of pain measurement. In: Pain Measurement and Assessment, R. Melzack (Ed.). New York: Raven Press, 1983.

9. Melzack, R., and P. Wall. Pain measurements: A new theory. Science 150:971-978, 1965.

10. Scott, J., and E.C. Huskisson. Graphic representation of pain. Pain 2:175-184, 1976.

11. Shellock, F.G. Effects of a topically applied counterirritant/analgesic on skin blood flow. Med. Sci. Sport Exer. 19:S49, 1987. (Abstract)

12. Talag, T. Residual muscle soreness as influenced by concentric, eccentric and static contractions. Res. Quar. 44:458-469, 1973.

13. White, J.R., and J.N. Sage. Topical analgesic on induced muscular pain. Phys. Ther. 50:166-172, 1970.

14. White, J.R., and J.N. Sage. Effects of a counterirritant on muscular distress in patients with arthritis.

Phys. Ther. 51:36-42, 1971. 